\title{
The long-term consequences of early-life dexamethasone treatment on the cognitive ability of male mice and gene expression in the hippocampus
}

\author{
U.I. Batluk ${ }^{1,2}$, K.V. Burdeeva ${ }^{3}$, A.O. Degtyareva ${ }^{1}$, O.M. Dolganova ${ }^{1,3}$, N.I. Ershov ${ }^{1}$, \\ T.I. Merkulova ${ }^{1,2}$, N.P. Bondar ${ }^{1,2 *}$ \\ ${ }^{1}$ Institute of Cytology and Genetics of SB RAS, Novosibirsk, Russia \\ ${ }^{2}$ Novosibirsk State University, Novosibirsk, Russia \\ ${ }^{3}$ Novosibirsk State Medical University, Novosibirsk, Russia \\ *e-mail:nbondar@bionet.nsc.ru
}

Key words: neonatal dexamethasone, transcriptome, hippocampus

Motivation and Aim: Dexamethasone (DEX) treatment in early life can activate HPA axis and lead to different negative consequences in adult ages.

Methods and Algorithms: DEX was injected on postnatal day 1-3 once a day with decreasing dose $(0.5,0.3,0.1 \mathrm{mg} / \mathrm{kg}$, s.c. $)$. Manifestation of neonatal reflexes and delayed effects on adult behavior were investigated. RNA-seq analysis in hippocampus was done on adult animals after neonatal treatment with DEX or saline.

Results: The DEX treatment in early life leads to delay in manifestation of neonatal reflexes and decrease in the weight of male mice. Adult mice that had received neonatal treatment with dexamethasone, showed a decreased anxiety and impaired spatial memory and learning in Morris water maze. To reveal the transcriptomic events in hippocampus accompanying the long-term behavioral effects of neonatal DEX treatment, an RNA-seq study was performed in adult mice. The expression of 12 genes was altered in DEXtreated mice compared to saline-treated animals. Among them, Arc gene seem to be the most appropriate to describe the observed cognitive disturbances, since it is tightly connected with formation of long-term memory as well as with regulation of synaptic plasticity.

Conclusion: Our data clearly demonstrated that neonatal treatment with DEX has adverse effects on cognitive abilities of adult mice. The transcriptomic study proposes only a few genes that may be involved in hippocampus malfunction, resulting in the observed cognitive impairments.

Acknowledgements: The work was supported by Russian Science Foundation project 16-15-10131. 